Ferrata Storti Foundation

\title{
Prognostic and predictive role of gene mutations in chronic lymphocytic leukemia: results from the pivotal phase III study COMPLEMENT1
}

\author{
Eugen Tausch, ${ }^{1}$ Philipp Beck, ${ }^{1}$ Richard F. Schlenk, ${ }^{1,2}$ Billy M.C. Jebaraj, ${ }^{1}$ \\ Anna Dolnik, ${ }^{1,3}$ Deyan Y. Yosifov, ${ }^{1,4}$ Peter Hillmen, ${ }^{5}$ Fritz Offner, ${ }^{6}$ Ann Janssens, ${ }^{7}$ \\ K. Govind Babu, ${ }^{8}$ Sebastian Grosicki, ${ }^{9}$ Jiri Mayer, ${ }^{10}$ Panagiotis Panagiotidis, ${ }^{11}$ \\ Astrid McKeown, ${ }^{12}$ Ira V. Gupta, ${ }^{13}$ Alexandra Skorupa, ${ }^{14}$ Celine Pallaud, ${ }^{15}$ \\ Lars Bullinger, ${ }^{1,3}$ Daniel Mertens, ${ }^{1,4}$ Hartmut Döhner ${ }^{1}$ and \\ Stephan Stilgenbauer ${ }^{1,16}$
}

Volume 105(10):2440-2447

\begin{abstract}
${ }^{1}$ Department of Internal Medicine III, UIm University, UIm, Germany; ${ }^{2}$ NCT-Trial Center, National Center for Tumor Diseases, German Cancer Research Center, Heidelberg, Germany; ${ }^{3}$ Klinik für Innere Medizin mit Schwerpunkt Hämatologie, Onkologie und Tumorimmunologie, Charité, Berlin; ${ }^{4}$ Mechanisms of Leukemogenesis, German Cancer Research Center (DKFZ), Heidelberg, Germany; ${ }^{5}$ Department of Haematology, St. James's University Hospital, Leeds, UK; 'Universitair Ziekenhuis Gent, Gent, Belgium; ${ }^{7}$ Universitair Ziekenhuis Leuven, Leuven, Belgium; ${ }^{8}$ Kidwai Memorial Institute of Oncology, Bangalore, India; ' Department of Hematology and Cancer Prevention, School of Public Health, Silesian Medical University in Katowice, Katowice, Poland; ${ }^{10}$ Department of Haematology-Oncology, University Hospital Brno, Brno, Czech Republic; ${ }^{11}$ University of Athens, Laikon General Hospital, Athens, Greece; ${ }^{12}$ Oncology Global Medicines Development, AstraZeneca, Melbourn, UK; ${ }^{13}$ GSK Oncology, GlaxoSmithKline, London, UK; ${ }^{14}$ Novartis Pharma GmbH, Nürnberg, Germany; ${ }^{15}$ Novartis AG, Basel, Switzerland and ${ }^{16}$ Department for Hematology, Oncology and Rheumatology, Saarland University Medical School, Homburg/Saar, Germany
\end{abstract}

\section{Correspondence:}

STEPHAN STILGENBAUER

stephan.stilgenbauer@uniklinik-ulm.de

Received: June 10, 2019.

Accepted: January 7, 2020.

Pre-published: January 9, 2020.

doi:10.3324/haematol.2019.229161

(C)2020 Ferrata Storti Foundation

Material published in Haematologica is covered by copyright. All rights are reserved to the Ferrata Storti Foundation. Use of published material is allowed under the following terms and conditions:

https://creativecommons.org/licenses/by-nc/4.0/legalcode. Copies of published material are allowed for personal or internal use. Sharing published material for non-commercial purposes is subject to the following conditions:

https://creativecommons.org/licenses/by-nc/4.0/legalcode, sect. 3. Reproducing and sharing published material for commercial purposes is not allowed without permission in writing from the publisher.

\section{ABSTRACT}

Text generation sequencing studies in chronic lymphocytic leukemia (CLL) have revealed novel genetic variants that have been associated with disease characteristics and outcome. The aim of this study was to evaluate the prognostic value of recurrent molecular abnormalities in patients with CLL. Therefore, we assessed their incidences and associations with other clinical and genetic markers in the prospective multicenter COMPLEMENT1 trial [treatment naive patients not eligible for intensive treatment randomized to chlorambucil (CHL) vs. ofatumumab-CHL $(\mathrm{O}-\mathrm{CHL})]$. Baseline samples were available from 383 patients $(85.6 \%)$ representative of the total trial cohort. Mutations were analyzed by ampliconbased targeted next generation sequencing (tNGS). In $52.2 \%$ of patients we found at least one mutation; the incidence was highest in NOTCH1 (17.0\%), followed by SF3B1 (14.1\%), ATM (11.7\%), TP53 (10.2\%), POT1 (7.0\%), RPS15 (4.4\%), FBXW7 (3.4\%), MYD88 (2.6\%), and BIRC3 (2.3\%). While most mutations lacked prognostic significance, TP53 (HR2.02, $P<0.01$ ), SF3B1 (HR1.66, $P=0.01$ ), and NOTCH1 (HR1.39, $P=0.03$ ) were associated with inferior progression-free survival (PFS) in univariate analysis. Multivariate analysis confirmed the independent prognostic role of TP53 for PFS (HR1.71, $P=0.04$ ) and overall survival (OS) (HR2.78, $P=0.02)$, and of SF3B1 for PFS only (HR1.52, $P=0.02$ ). Notably, NOTCH1 mutation status separates patients with a strong from those with a weak benefit from addition of ofatumumab to CHL (NOTCH $1^{\text {wt }}$ : HR0.50, $P<0.01$; NOTCH ${ }^{\text {mut }}$ : HR0.81, $\left.P=0.45\right)$. In summary, TP53 and SF3B1 were confirmed as independent prognostic factors and NOTCH1 as a predictive factor for reduced ofatumumab efficacy in a randomized chemo/immunotherapy CLL trial. These results validate NGS-based mutation analysis in a multicenter trial and provide a basis for expanding molecular testing in the prognostic workup of patients with CLL. (Trial registered at clinicaltrials.gov identifier: NCT00748189.) 


\section{Introduction}

Chronic lymphocytic leukemia (CLL) is a heterogeneous disease. Many prognostic factors have been identified in the last decades, but only a few have found their way into clinical practice. ${ }^{1}$ In a variety of different CLL trials, genomic aberrations, particularly $17 \mathrm{p}$ deletion and mutation status for TP $53^{2-7}$ had the strongest relation to clinical outcome. Their assessment before institution of therapy has therefore been recommended for every CLL patient. ${ }^{1}$ The availability of new CLL treatment modalities have raised the question of the influence of these genomic aberrations as predictive factors for treatment response.

Recent insights from unbiased next generation sequencing (NGS) approaches describe more than 40 recurrently mutated cancer driver genes in CLL, the clinical significance of which often remains undefined..$^{8 \cdot 11}$ The clinical value of single mutations was mostly studied in heterogeneous patient cohorts and assessed outside of the context of clinical and biologic features. ${ }^{12-16}$ Therefore, there is a need to perform mutation screening in large clinical trials, as they provide a homogeneous cohort of patients, standardized assessment of clinical and laboratory parameters, and valid outcome data. The UK CLL4 trial and the GCLLSG CLL8 trials served to determine the prognostic impact of recurrent mutations among the minority of young/fit patients. Both cohorts were screened for mutations in TP53, NOTCH1 and SF3B1, as mutations in these genes have a high incidence (10-15\%) and typically occur in hotspots, which allows the use of Sanger sequencing for mutation screening. While mutated TP53 (TP53 ${ }^{\text {mut }}$ ) was validated as an independent prognostic factor for progression-free survival (PFS) and overall survival (OS) in both studies, results for the other genes were less clear: mutated SF3B1 (SF3B $\left.1^{\mathrm{mut}}\right)$ was associated with decreased PFS in CLL8 and decreased OS in UK CLL4 but not vice versa. ${ }^{12,13,17}$ Notably, and in contrast to previous reports, mutated NOTCH1 (NOTCH ${ }^{\text {mut }}$ ) was not an independent prognostic factor for PFS in both trials, but was identified as predicting the lack of efficacy of the addition of CD20 antibody (rituximab) in the CLL8 trial $i^{13}$ this provocative finding needs confirmation in additional data sets.

The COMPLEMENT1 trial evaluated chlorambucil (CHL), a less intense chemotherapy backbone for which the majority of CLL patients are eligible, with or without the addition of the CD20 antibody ofatumumab. ${ }^{15,16}$ Ofatumumab binds to a different epitope of CD20 compared to rituximab and is more efficacious in triggering complement-dependent cytotoxicity (CDC) in vitro. The major result of COMPLEMENT1 was a significantly prolonged PFS by the addition of ofatumumab (22.4 months; 95\% CI: 19.0-25.2) to chlorambucil ${ }^{18,19}$ (13.1 months; $95 \%$ CI: $10.6-13.8)$ in a population who cannot tolerate more intensive therapy. The well characterized patient cohort and mature follow up of COMPLEMENT1 provided an ideal background to study incidence, associations, and prognostic as well as the predictive value of gene mutations in a typical, elderly, front-line CLL patient population.

\section{Methods}

\section{Patients}

The GlaxoSmithKline-sponsored phase III trial COMPLEMENT1 (OMB110911) enrolled 447 untreated patients ineligible for fludarabine-based therapy and in need of treatment. Subjects were randomly assigned to receive $\mathrm{CHL}$ or $\mathrm{O}-\mathrm{CHL}$ with a ratio of 1:1. The primary study end point was PFS as determined by an independent review committee. Biomarker analysis was implemented in the study protocol and was approved by the institutional review board or ethics committee of each participating institution. Each patient provided written informed consent before enrolment. For 383 of 447 patients (85.7\%), a baseline sample with sufficient DNA and informed consent for research purposes were available. This subset was representative of the intent-to-treat population with regard to clinical, laboratory and genetic baseline characteristics (Online Supplementary Table S1).

\section{Sequencing}

We designed a customized Illumina ${ }^{\mathrm{TM}}$ Truseq amplicon panel for all coding regions of TP53, ATM, BIRC3, MYD88, FBXW7, POT1, and for the most commonly affected exons 14, 15, 16 and 18 in SF3B1 and exon 34 in NOTCH1. The cumulative target size was 24,161 basepairs (bp) covered by 221 amplicons with a length of up to $250 \mathrm{bp}$ each. Adjacent 10 intron bp were included to cover splice site mutations. Input of $250 \mathrm{ng}$ DNA from peripheral blood mononuclear cells isolated by Ficoll gradient centrifugation were sufficient for libraries according to the Illumina TruSeq Custom Amplicon (TSCA) protocol. Sequencing was performed on an Illumina MiSeq ${ }^{\mathrm{TM}}$ with the $500-$ cycle MiSeq Reagent Kit v2. Mutations in the 3'UTR of NOTCH1 and in RPS15 exon 4 were analyzed via Sanger sequencing.

\section{Statistical analysis}

Statistical analyses were performed on an intent-to-treat basis including all patients with samples available. Analysis was performed using R studio 1.1.447 with survival package (RStudio Inc., Boston, MA, USA). Categorical variables were compared using the Fisher Exact test, and continuous variables were compared using non-parametric rank-sum tests. Statistical tests were two-sided; $P<0.05$ was considered statistically significant. There were no adjustments for multiple testing, so that all reported $P$ values have an exploratory character for all analysis except for Online Supplementary Figure S2 for which we used false discovery rate (FDR) as an adjustment.

Time to event was analyzed by Kaplan-Meier estimates and log-rank. To identify independent prognostic factors, we included treatment arm, del11q, del17p and IGHV mutation status as factors associated with PFS in univariate analysis in addition to all genetic subgroups defined by gene mutations. These 13 parameters were tested in 383 patients without forward or backward selection procedures to explore the independent prognostic character. In addition, we performed a multivariate treatment-gene mutation interaction analysis to explore treatment dependent and therefore predictive value. We used $\mathrm{R}$ studio 1.1.447 (RStudio Inc., Boston, MA, USA) with survival package for all statistical analyses.

\section{Results}

\section{Spectrum and associations of mutations}

Sequencing of 383 patient samples resulted in a mean coverage of 2,852 reads and $98.2 \%$ of target reads above 100x. We identified a total of 304 mutations in the selected gene set. Mutation incidences were NOTCH1 (17.0\%), SF3B1 (14.1\%), ATM (11.7\%), TP53 (10.2\%), POT1 (7.0\%), RPS15 (4.4\%), FBXW7 (3.4\%), MYD88 (2.6\%), and BIRC3 $(2.3 \%)$ (Figure 1A). When looking at the muta- 

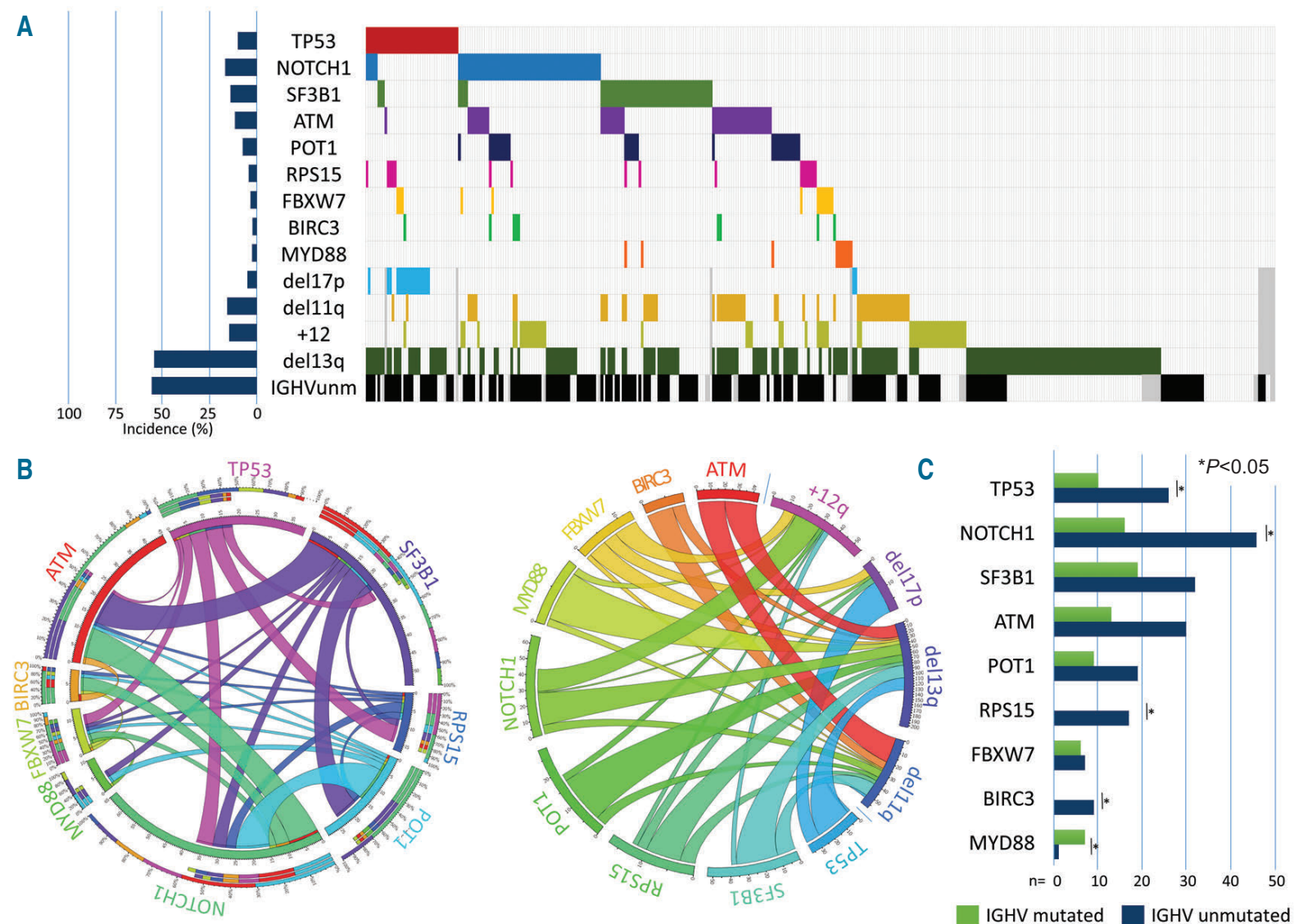

IGHV mutated IGHV unmutated

Figure 1. Incidence and distribution of genetic parameters (gene mutations, genomic aberrations and IGHV status). (A) Cluster diagram of patients (columns) with data for all genetic parameters (rows) (right) and overall incidence (left). Distribution of markers is ordered by rows. (B) Circos plots of the co-occurrence of gene mutations with each other (left) and pairwise with chromosomal aberrations (right). Lengths of arcs correspond to total incidences of respective markers while the width of each ribbon corresponds to the proportion of co-occurrence with a respective second marker. (C) Distribution of gene mutations in the IGHV mutation status subgroups.

tion type, we observed 195 missense mutations, 3 insertions, 56 deletions, 27 nonsense mutations, 11 splice site and 12 3'UTR mutations. Of all variants, 122 were selected for validation via Sanger sequencing and all of them were confirmed, so that further validation procedures were omitted (Online Supplementary Table S2). While the number of mutations was slightly higher in CHL versus O-CHL (178 vs. 126, not significant), the number of patients with at least one mutation was similar in both arms: $55 \%$ for CHL and $53 \%$ for O-CHL (see Online Supplementary Figure $S 1$ for a detailed overview). In the total cohort, only $11.5 \%$ of patients had neither a mutation nor a chromosomal aberration in the analyzed targets. As previously reported, mutations in SF3B1 and MYD88 were exclusively single nucleotide variants (SNV). Of 66 NOTCH1 mutations, 52 caused stop codons via frameshift or nonsense mutation and 12 affected the 3'UTR (Online Supplementary Table S2). TP53 and BIRC3 showed various patterns of mutations including insertions, deletions and exonic as well as splice site SNV.

Regarding associations, all but one case with MYD88 mutation had mutated IGHV, while TP53, RPS15 and BIRC3 mutations were found predominantly in patients with unmutated IGHV (Figure 1). As to cytogenetics, we found deletion of $11 \mathrm{q}$ associated with mutations of ATM and BIRC3 and trisomy $12 \mathrm{q}$ associated with mutations in
NOTCH1, BIRC3 and FBXW7. Interestingly, of the nine BIRC3 mutated cases, six had an aberration in $11 \mathrm{q}$ and four in 12q. As expected, high concordance was found between $17 \mathrm{p}$ deletion and mutation in TP53, but also with $F B X W 7^{\text {mut }}$. These associations hold true when considering correction for multiple testing with false discovery rate (Online Supplementary Figure S2).

Analyzing mutations in nine different genes and correlation with genomic aberrations and IGHV mutation status allowed us to derive an interaction network based on significant correlations considering incidence, co-occurrence, or mutual exclusivity (Figure 2). Interestingly, two dichotomies emerge from the complex network. Firstly, there is a mutual exclusivity between del13q and $+12 q$. While del13q does not significantly associate with any of the gene mutations, cases with +12 q cluster with mutated NOTCH1, BIRC3 and FBXW7. The second dichotomy is found in mutation of TP53 and del17p on one side and a cluster including del11q, $+12 \mathrm{q}$ and $A T M$ on the other side. Interestingly, mutations of SF3B1 are not associated with either group, suggesting that these mutations represent an independent pathogenic mechanism. Concerning clinical associations, SF3B1 mutations were more common in male patients (Online Supplementary Table S3) and associated with high absolute lymphocyte count (ALC) and $\mathrm{CD} 19^{+} \mathrm{CD}^{+}$fraction by flow cytometry $(P<0.01$ and 
Table 1. Prognostic associations of gene mutations and progression-free survival (PFS) / overall survival (OS) in the full trial cohort (both treatment arms combined) in univariate analysis (log rank test).

\begin{tabular}{l|ccc|ccc|c} 
Gene mutation & HR & $\begin{array}{c}\text { PFS } \\
\text { 95\%CI }\end{array}$ & $\mathbf{P}$ & HR & 95\%CI & P & N (events PFS/0S) \\
TP53 & 2.02 & $1.18-3.45$ & $<\mathbf{0 . 0 1}$ & 4.25 & $1.65-10.92$ & $<\mathbf{0 . 0 1}$ & $39(27 / 16)$ \\
NOTCH1 & 1.39 & $1.04-1.86$ & $\mathbf{0 . 0 3}$ & 0.84 & $0.49-1.44$ & 0.62 & $65(50 / 10)$ \\
\hline SF3B1 & 1.66 & $1.12-2.47$ & $<\mathbf{0 . 0 1}$ & 1.39 & $0.67-2.87$ & 0.32 & $54(43 / 11)$ \\
ATM & 1.16 & $0.84-1.60$ & 0.42 & 0.89 & $0.48-1.68$ & 0.78 & $45(34 / 7)$ \\
\hline POT1 & 1.25 & $0.73-1.92$ & 0.33 & 1.15 & $0.50-2.58$ & 0.77 & $29(21 / 5)$ \\
RPS15 & 1.08 & $0.62-1.87$ & 0.81 & 2.06 & $0.72-5.93$ & 0.11 & $17(10 / 5)$ \\
\hline FBXW7 & 1.51 & $0.67-3.39$ & 0.22 & 2.19 & $0.52-9.33$ & 0.12 & $13(9 / 4)$ \\
MYD88 & 0.59 & $0.31-1.11$ & 0.19 & NA & NA & NA & $10(6 / 0)$ \\
\hline BIRC3 & 1.63 & $0.69-3.87$ & 0.23 & 1.29 & $0.34-4.92$ & 0.72 & $9(6 / 2)$ \\
\hline
\end{tabular}

NA: not applicable due to no events in mutated patients. CI: Confidence Interval; N: number. Statistical significance in bold.

$P=0.03)$, whereas ALC in MYD88 mutated patients was low. WBC $>50 \times 10^{9} / \mathrm{L}$ were found more often in patients with mutated TP53, RPS15 or ATM $(P=0.05, P=0.02$ and $P=0.03$, respectively).

\section{Clinical outcome and prognostic impact of gene mutations in the full cohort}

A significantly lower overall response rate was found only for TP53 mutated cases (HR 5.20, P<0.01). In univariate analysis, significantly decreased PFS was found for patients with mutations in TP53 (HR 2.02, $P<0.01$ ), SF3B1 (HR 1.66, $P<0.01$ ), and NOTCH1 (HR 1.39, $\mathrm{P}=0.03$ ), but not for patients with mutations in ATM (HR 1.16, $P=0.42)$ or BIRC3 (HR 1.63, $P=0.23$ ) (Table 1 with 95\%CI, Figure 3 , and Online Supplementary Figure S3). OS was significantly shorter in patients with mutations of TP53, and this was observed both in the total cohort (HR 4.25, $P<0.01$ ) as well as in patients without deletion of 17p (HR 2.56, $P=0.03$ ) (data not shown). Remarkably, no death event was observed among MYD88 mutated patients. The number of mutated genes correlated with PFS, and patients harboring two or more mutated genes had shorter PFS (Online Supplementary Figure S4). Mutation in at least one of the target genes associated with significantly shorter OS, while the number of mutated genes did not play a significant role.

Taking into account that a disruption of both alleles of BIRC 3 or ATM could be required to observe any effect as described in the UK CLL ${ }^{20}$ trial, we considered the del11q status for the impact of mutations in both genes. However, in our patient cohort, mutation of ATM did not add significant prognostic value either in the 11q deleted subgroup or in the $11 \mathrm{q}$ disomic subgroup (Online Supplementary Figure S5). As BIRC3 was mutated in only nine patients, the subgroups were too small to address this question.

Mutations in TP53 and ATM showed high variant allelic fractions (VAF) of the mutant allele, mainly explained by a high co-occurrence with deletion of the other allele (Online Supplementary Figure S6A). Also mutations with a variant allele fraction (VAF) $\leq 10 \%$, usually undetectable by Sanger sequencing, were rare in TP53 (2 of 38) and absent in MYD 88 in this trial, but present in a significant proportion of cases with mutation of SF3B1, NOTCH1 or $F B X W 7$. As the role of mutations with minor allelic fraction is still unclear, we performed survival analyses including patients with wild-type (WT) SF3B1/NOTCH1 and with major and minor $(<10 \%$ VAF) mutated fractions (only NGS data considered). Interestingly, patients affected by only minor SF3B1 mutated clones showed significantly shorter PFS in comparison to WT (HR 3.09, 95\% CI: $0.67-14.30, P=0.01$ ), while for NOTCH1, minor mutations had no significant impact on the full cohort, mainly due to low numbers (HR 1.54, 95\% CI: 0.71-3.34, $P=0.26$ ) (Online Supplementary Figure S6B and C).

We performed multivariable analyses including treatment arm, IGHV mutation status, del17p, del11q and gene mutations to examine the independent prognostic value of these parameters. Besides ofatumumab+chlorambucil, IGHV status, del17p and del11q, only mutations of TP53 (HR 1.71, $P=0.04$ ) and SF3B1 (HR 1.52, $P=0.02$ ) were identified as independent factors associated with decreased PFS. For OS, only presence of del17p and TP53 mutation (HR 2.78, $P=0.02$ ) retained a significant independent role (Table 2 with $95 \% \mathrm{CI}$ ).

\section{Predictive value for the efficacy of ofatumumab addition}

Randomization of patients into treatment arms with and without ofatumumab allowed the evaluation of differential CD20 antibody efficacy in subgroups defined by mutations. In the total cohort of 383 patients, treatment with ofatumumab was beneficial with regard to PFS (HR $0.53, P<0.01)$ as published for the whole trial population. ${ }^{19}$ Analyses of both treatment arms separately generally reiterated the results obtained for the total cohort, with mutations in SF3B1 and TP53 being associated with shorter PFS. However, for NOTCH1, we observed an impact on PFS in the O-CHL treatment arm (HR 1.94, 95\%CI: $1.25-$ 3.92, $P<0.01$ ) but not with CHL alone (HR 1.01, 95\% CI: $0.69-1.47, P=0.98$ ) (Figure 4). Conversely, the addition of ofatumumab to chlorambucil in patients with $\mathrm{NOTCH}{ }^{\text {wt }}$ status was strongly beneficial (mPFS 23.8 vs. 13.3 months, HR 0.50 , 95\%CI: $0.39-0.63, P<0.01$ ), while the benefit in the NOTCH $1^{\text {mut }}$ group was not significant (17.2 vs. 13.1 months, HR 0.81, 95\% CI: 0.50-1.31, $P=0.45)$. Notably, the same analysis confirmed the addition of ofatumumab to be beneficial in SF3B1 and TP53 mutated subgroups (for SF3B1: mPFS 17.3 vs. 10.8 months, HR 0.53 , 95\% CI: 0.29 0.97, $P=0.03$; for TP53: mPFS 12.8 vs. 3.7 months, HR 0.49, 95\% CI: $0.23-1.05, P=0.05)$. This impact was strongest with NOTCH1 mutations at a mutant allele fraction $>40 \%$ and weaker with smaller NOTCH1 variant fraction (Online Supplementary Figure S7). To investigate the rela- 


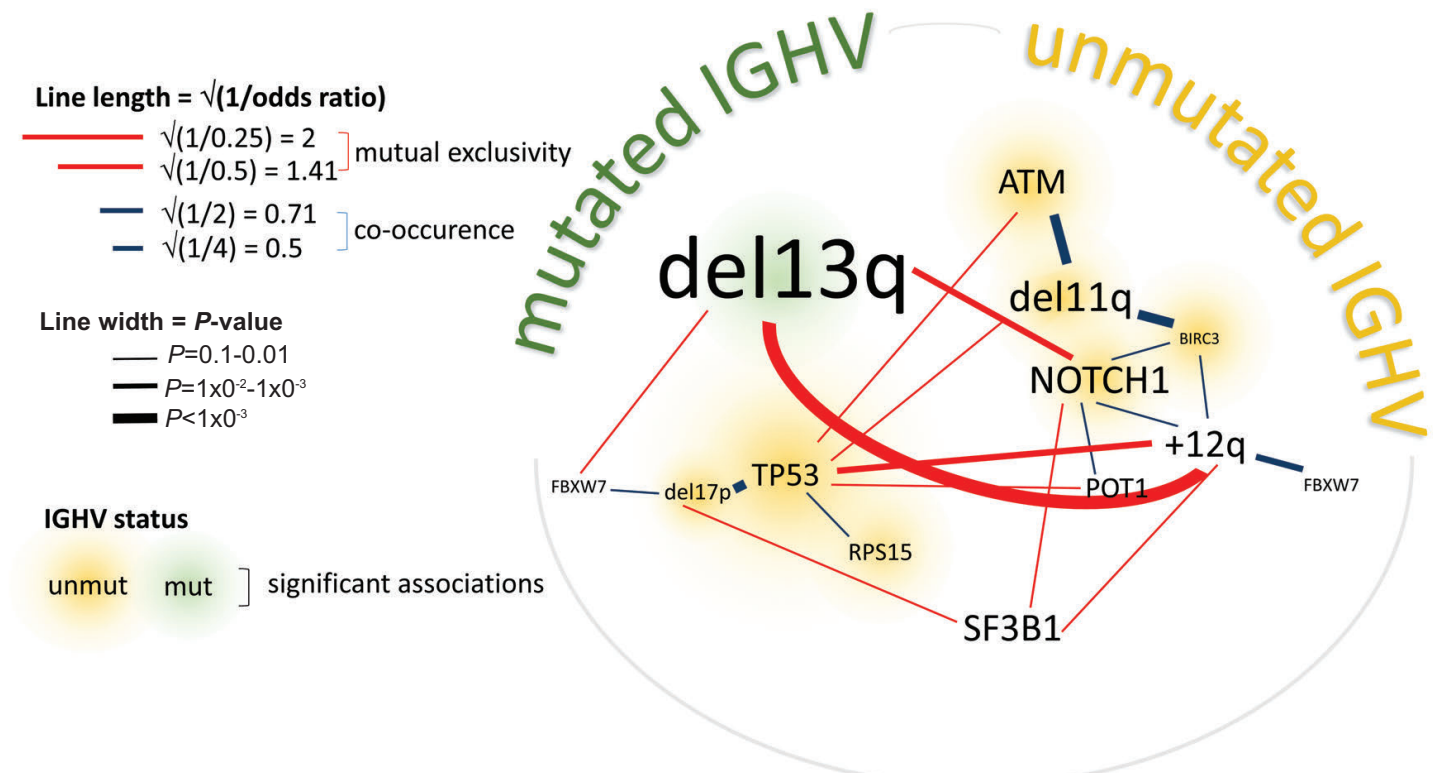

Figure 2. Visualization of co-occurrence of gene mutations and genomic aberrations based on pairwise Fisher exact test. Line length corresponds to $\sqrt{ }(1 /$ odds ratio) Therefore, lines with a length $>1$ show mutual exclusivity (red) and lines with a length $<1$ co-occurrence (blue). Line width corresponds to stated $P$-value of pairwise comparison; when $P>0.1$, no line is depicted. Font size characterizes incidence of mutation/aberration; green indicates association with mutated IGHV, and yellow indicates association with unmutated IGHV $(P<0.1$ each). FBXW7 is depicted twice.

tion between treatment and NOTCH1 mutational status, we performed an interaction-focused multivariate test. This analysis attributed a predictive impact to NOTCH1 $(P=0.05)$, while for all other parameters no interaction was observed (Online Supplementary Table S4). There were no differences in CD20 surface levels measured with flow cytometry between subgroups defined by mutation of NOTCH1 suggesting that differential CD20 expression is not the cause of the lower CD20 antibody efficacy (data not shown).

\section{Discussion}

Over recent years, in an increasing number of laboratories, tNGS has replaced Sanger sequencing as a tool for mutation analysis. The current report represents a combined analysis of recurrent gene mutations studied by tNGS with a comprehensive dataset in a large multicenter phase III trial in CLL. In contrast to prior analyses, we used amplicon-based tNGS which allowed sequencing of 119 exons in eight different genes with a detection limit of $5 \%$ VAF. Although some tNGS approaches allow detection of subclones with only $1 \%$ VAF and below, ${ }^{21}$ the aim of this project was to evaluate the prognostic value of molecular abnormalities for a better refinement of risk stratification. Development of such a robust, reproducible and affordable assay, which can be easily adopted into a diagnostic setup, could help in the clinical management of CLL patients. In favor of a uniform coverage of all targets, we trimmed sensitivity to achieve reliable results with high specificity. Mutations with minor allelic fractions are not detectable via Sanger sequencing and there is little evidence of their clinical impact, apart from in TP53., ${ }^{21,22}$ Therefore, not surprisingly, councils like the European Initiative for Research in CLL (ERIC) caution against deriving clinical conclusions from small subclonal mutations. ${ }^{23}$
The low number of detected minor mutations in TP53 and other genes in COMPLEMENT1 does not allow any conclusions to be drawn about their prognostic significance. However, for minor SF3B1 mutations, we found an impact similar to mutations with VAF $>10 \%$. Further studies on clinical trials focusing on minor mutations are necessary to explore the impact of such variants with a comprehensive validation setup.

Our data support prior observations characterizing SF3B1, NOTCH1, ATM and TP53 as the most frequently mutated genes in decreasing order of frequency, while mutations in BIRC3 and MYD88 remain very rare events in untreated CLL. ${ }^{10,11}$ However, putting associations of these mutations and chromosomal aberrations in a correlation network uncovers two substantial associations. First, the mutual exclusivity between del13q and $+12 q$ and associated mutations, which is supported by prior clonality and lineage analysis showing that both aberrations are clonal events in CLL associating with tumor pathogenesis, but independently of each other. ${ }^{10}$ Second, intermediate events, namely del17p and TP53 on one side and different genomic abnormalities, mainly connected to $11 \mathrm{q}$ and $A T M$ on the other side. As these abnormalities associate with growth advantage and accumulation in pretreated CLL, one can envisage that either mutation of TP53 or mutation of one of the other genes is sufficient to cause a clonal survival advantage and therefore make the respective other event redundant.

Besides genetic interactions, analysis of the impact on clinical parameters, and especially on outcome after therapy, was the major aim of this study. In the current COMPLEMENT1 data set, TP53 was the only gene mutation associated with a decrease in PFS and OS independent of other prognostic factors in multivariable analyses. Notably, this correlation was observed despite the high degree of collinearity with $17 \mathrm{p}$ deletion, confirming the need of TP53 mutation testing in addition to $17 \mathrm{p}$ deletion 

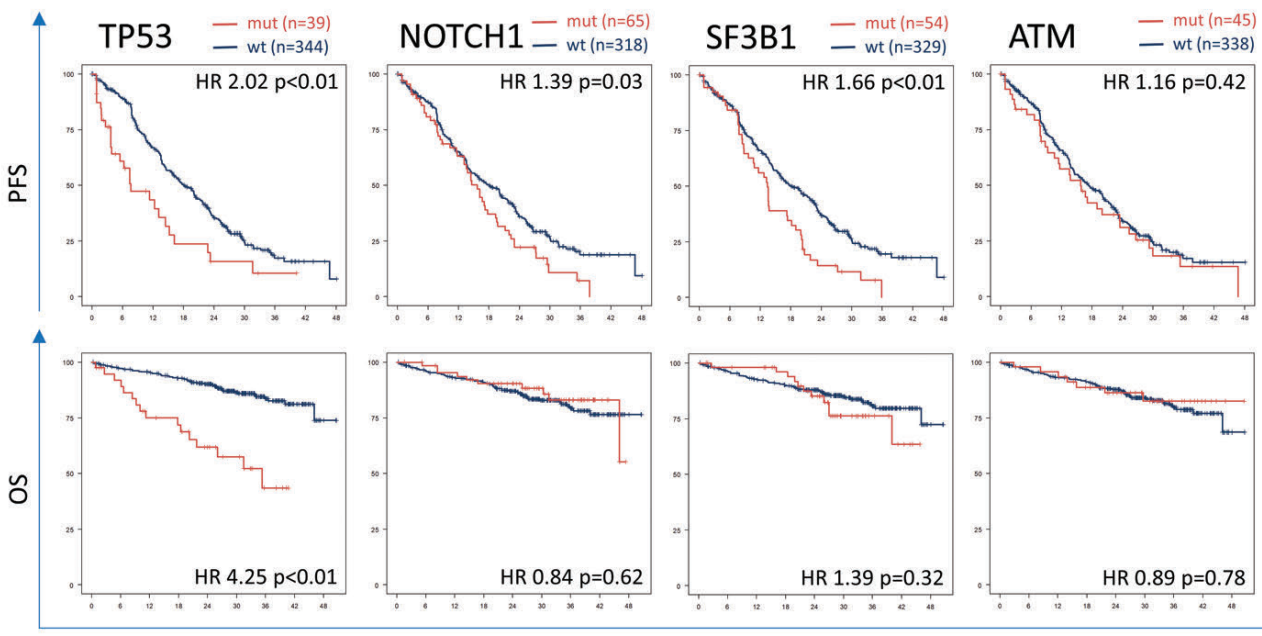

BIRC3 二 mut $(n=9)_{\text {wt }(n=374)}$

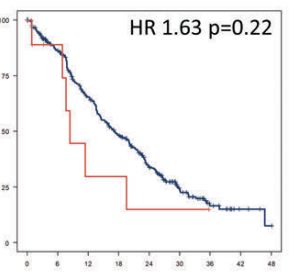

$n=383$ for all analyses
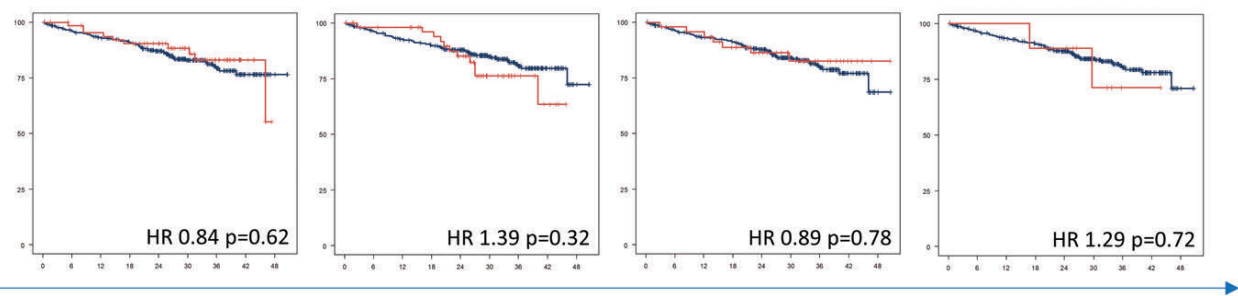

months

Figure 3. Kaplan-Meier estimates of progression-free survival (PFS) (left) and overall survival (OS) (right) according to the status of selected gene mutations for the total patient cohort. Red lines: mutated (mut) subgroups; blue lines: wild-type (wt). Denoted $P$-values were calculated by log-rank test (mut vs. unmutated subgroup).

Table 2. Multivariate analysis based on Cox-regression for progression-free survival (PFS) / overall survival (OS).

\begin{tabular}{|c|c|c|c|c|c|c|c|}
\hline Parameter present & HR & $\begin{array}{l}\text { PFS } \\
95 \% \text { CI }\end{array}$ & $P$ & HR & $\begin{array}{c}\text { OS } \\
95 \% \mathrm{CI}\end{array}$ & $\boldsymbol{P}$ & N (events PFS/OS) \\
\hline O-CHL arm & 0.47 & $0.35-0.61$ & $<0.01$ & 0.76 & $0.44-1.29$ & 0.31 & $183(116 / 28)$ \\
\hline TP53 & 1.71 & $1.04-2.81$ & 0.04 & 2.78 & $1.17-6.62$ & 0.02 & $39(27 / 16)$ \\
\hline NOTCHI & 1.32 & $0.95-1.84$ & 0.10 & 0.88 & $0.43-1.81$ & 0.74 & $65(50 / 10)$ \\
\hline$S F 3 B 1$ & 1.52 & $1.06-2.17$ & 0.02 & 1.31 & $0.62-2.8$ & 0.48 & $54(43 / 11)$ \\
\hline ATM & 1.02 & $0.67-1.56$ & 0.93 & 1.01 & $0.43-2.38$ & 0.98 & $45(34 / 7)$ \\
\hline POTI & 1.28 & $0.79-2.1$ & 0.32 & 1.65 & $0.62-4.4$ & 0.31 & $29(21 / 5)$ \\
\hline RPS15 & 0.82 & $0.42-1.59$ & 0.56 & 1.16 & $0.44-3.06$ & 0.76 & $17(10 / 5)$ \\
\hline$F B X W 7$ & 1.15 & $0.58-2.29$ & 0.69 & 1.64 & $0.56-4.83$ & 0.37 & $13(9 / 4)$ \\
\hline MYD88 & 0.72 & $0.29-1.78$ & 0.47 & $\mathrm{NA}$ & NA & $\mathrm{NA}$ & $10(6 / 0)$ \\
\hline BIRC3 & 1.12 & $0.47-2.66$ & 0.79 & 0.63 & $0.15-2.69$ & 0.53 & $9(6 / 2)$ \\
\hline IGHVunut & 1.46 & $1.09-1.95$ & 0.01 & 1.72 & $0.95-3.13$ & 0.08 & $199(141 / 44)$ \\
\hline del17p & 2.90 & $1.49-5.67$ & $<0.01$ & 3.22 & $1.18-8.76$ & 0.02 & $19(12 / 10)$ \\
\hline dell1g & 1.72 & $1.19-2.51$ & $<0.01$ & 1.94 & $0.98-3.83$ & 0.06 & $58(46 / 14)$ \\
\hline
\end{tabular}

NA: not applicable due to no events in mutated patients. CI: Confidence Interval; $\mathrm{N}$ : number. Statistical significance in bold.

diagnostics in routine practice, as recommended by current ERIC and International Workshop on CLL (iwCLL) guidelines. ${ }^{1}$ Accordingly, as only about half of the TP53 mutated cases harbor an additional 17p deletion, mutation status for TP53 should be assessed in addition to del17p to allocate patients to treatment with novel agents. However, this subgroup accounts for just over 10\% (here $11.7 \%$ ) of front-line CLL cases, and does not identify all patients with poor response and short survival times. Therefore, the major question was whether other genetic factors characterize aggressive CLL to the same degree. In previous studies, this had been shown for disrupted $B I R C 3$, that affected the response to therapy and PFS in an extent comparable to mutation of TP53. ${ }^{24}$ In COMPLEMENT1, only nine patients were mutated in BIRC3, having an overall response rate (ORR) of $75 \%$ in contrast to $47 \%$ responders among TP53 mutated cases. While TP53 significantly shortened PFS (HR 2.02, $P<0.01)$ and OS (HR
4.25, $P<0.01$ ), BIRC3 did not associate with either (HR 1.63 for PFS and 1.29 for OS, both not significant). The same applies to mutations in ATM, which together with mutations in BIRC3 associate with del11q, but do not add any additional prognostic value to the impact of $11 \mathrm{q}$ on PFS and OS in this trial. This is in contrast to data published within the UK CLL4 trial, ${ }^{20}$ which may be explained by a different treatment and the lack of a CD20 antibody in UK CLL4, a different filtering approach for ATM variants, or just smaller patient numbers. However, also other groups did not find an adverse impact of a biallelic inactivation in $A T M,{ }^{25,26}$ and therefore a more comprehensive setup with a bigger patient cohort with matched germline samples for validation of the somatic origin of these variants is required to definitively answer this question.

In contrast, for mutations of $S F 3 B 1$, we observed a higher risk for early progression independently from other prognostic factors, but this was less pronounced as com- 
$\mathrm{O}-\mathrm{CHL}$

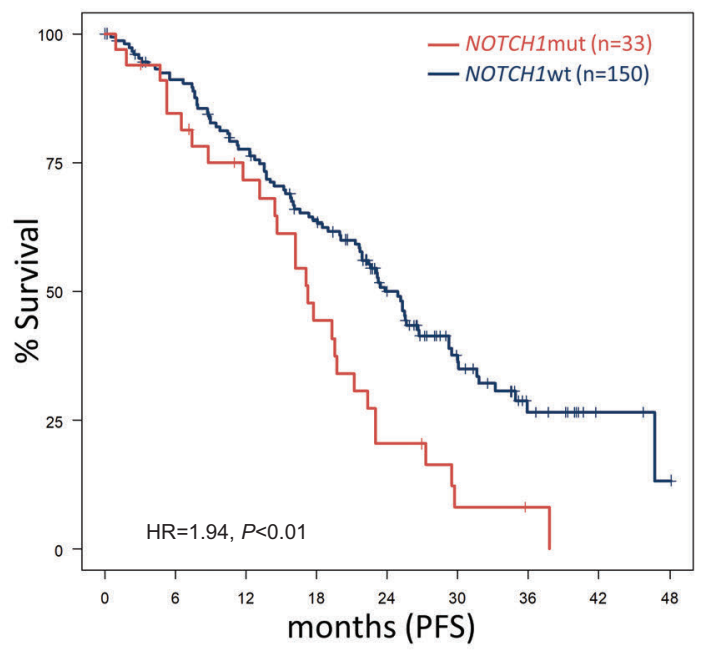

$\mathrm{CHL}$

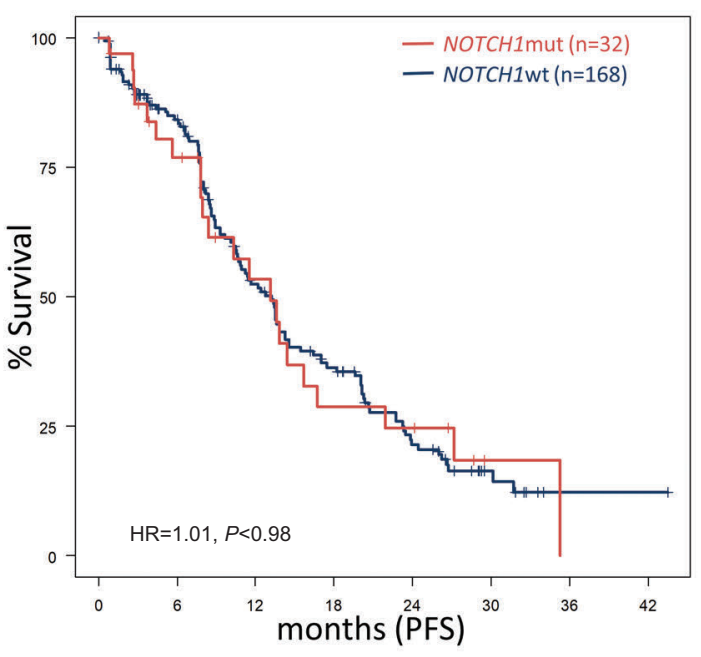

Figure 4. Kaplan-Meier estimates of progression-free survival (PFS) in the chlorambucil + ofatumumab (left) and chlorambucil alone (right) treatment arms according to NOTCH1 mutation status. Mutated subgroups are depicted by red lines, wild type by blue lines. Denoted P-values were calculated by log-rank test (mutated vs. unmutated subgroup).

pared to TP53 or del17p. These results conform to a number of previous observations. ${ }^{8,13,27,28}$ Some approaches integrated $S F 3 B 1$ and additional novel mutations into the hierarchical classification for prognostication established by Döhner in 2000. ${ }^{3}$ This is challenging as coincidences like mutations of NOTCH1 and $+12 \mathrm{q}$ or ATM and del11q are not easy to resolve in these models and currently available data are not powered to address correlations of small subgroups with clinical parameters. In a recent analysis, del11q and mutations of SF3B1 and NOTCH1 were categorized as intermediate risk. ${ }^{29}$ Although del11q and SF3B1 mutation showed similar hazard ratios for PFS $(1.72$ and $1.52)$ in the multivariate analysis of COMPLEMENT1, the former shows a trend to inferior $\mathrm{OS}$ (HR 1.94, $P=0.06$ ) while $S F 3 B 1^{\text {mut }}$ did not (HR 1.31, $P=0.48$ ). In addition, the prognostic impact of NOTCH1 appeared to strongly depend on the type of therapy (see below and Stilgenbauer et al. ${ }^{13}$ ), indicating that its use as a prognostic marker must be approached with caution.

As in previous genomic studies, we observed an adverse outcome of NOTCH $1^{\text {mut }}$ patients on the total trial cohort, which is more pronounced in cases with coding mutations. However, when analyzing both treatment arms separately, this prognostic impact turned out to be due to differential treatment effect, i.e. it identified NOTCH1 mutation as a predictive factor. This finding reiterates the result obtained with rituximab, confirming a lesser efficacy of type 1 CD20 antibodies in NOTCH1 mutated $\mathrm{CLL}^{13}$ compared to WT. The impact on PFS is strongest in patients with a high mutant allele burden in NOTCH1 and less pronounced in patients with minor mutations, but can only be observed with $\mathrm{O}-\mathrm{CHL}$ and not with CHL treatment. This predictive value remains after adding 3'UTR mutations in NOTCH1 and persisted in a treatment interaction analysis in contrast to all other markers. This may explain the discrepancies in previously published data: mutation of NOTCH1 was an independent prognostic factor for PFS in heterogeneous cohorts of patients mainly treated with CD20 antibodies as the current standard of therapy but was not in the UK LRF CLL4 trial that did not contain CD20 antibody treatment. ${ }^{12,14,15,30,31}$ Interestingly, in contrast to the previously published relation between NOTCH1 mutation and CD20 expression, ${ }^{32}$ NOTCH $1^{\text {mut }}$ cases showed no difference in CD20 surface expression as analyzed by flow cytometry. This is in line with the results from the CLL8 trial but differs from recently published data. ${ }^{13,32}$ Even though the underlying molecular interrelation of NOTCH1 mutations with CD20 remains unclear, the predictive impact of NOTCH1 mutation is now confirmed in two independent clinical phase III trial cohorts. Although $\mathrm{O}-\mathrm{CHL}$ shows slightly longer mPFS than CHL in NOTCH $1^{\text {mut }}$ patients, it is noteworthy that both mutation subgroups, NOTCH $1^{\text {mut }}$ and TP5 $3^{\text {mut }}$, have a similar initial impact in the setting of chemo-immunotherapy with CD20-targeted therapy outcome (HR on PFS 1.88 for $T P 53^{\text {mut }}$ and 1.94 for NOTCH $\left.1^{\text {mut }}\right)$. Moreover, the proportion of patients affected by mutated NOTCH1 in the front-line setting is bigger than the group defined by TP53 mutation. Based on current treatment guidelines, chemoimmunotherapy is still a valuable option for a number of patients. ${ }^{33}$ Our findings raise a note of caution on the use of such therapy in a significant subset of CLL patients with mutated NOTCH1. Furthermore, CD20 antibodies remain important elements in combination with novel compounds e.g. with venetoclax ${ }^{34}$ or ibrutinib, ${ }^{35}$ and it is still unclear whether specific subgroups have a particular benefit of such combinations in comparison to single agent. The major implication from our observation is the need to understand and circumvent the resistance against CD20 antibodies as they remain an important element in the treatment of CLL. Furthermore, our results underline the role of recurrent mutations also in trials with novel treatment principles such as BTK-, PI3K- and BCL2 inhibition.

\section{Acknowledgments}

The authors thank all patients, their families and their physicians for trial participation and donation of samples. The authors thank Simon Müller for supporting experiments. 


\section{Funding}

This work was supported by the Else Kröner-FreseniusStiftung (2010_Kolleg24), EC (01KT1601, FIRE CLL), BMBF
(031L0076C PRECISe), and Deutsche Forschungsgemeinschaft (SFB 1074 projects B1, B2). Genetic analyses were supported by GlaxoSmithKline and Novartis.

\section{References}

1. Hallek M, Cheson BD, Catovsky D, et al. iwCLL guidelines for diagnosis, indications for treatment, response assessment, and supportive management of CLL. Blood. 2018;131(25):2745-2760

2. Zenz T, Eichhorst B, Busch R, et al. TP53 mutation and survival in chronic lymphocytic leukemia. J Clin Oncol. 2010; 28(29):4473-4479

3. Döhner H, Stilgenbauer S, Benner A, et al. Genomic Aberrations and Survival in Chronic Lymphocytic Leukemia. N Engl J Med. 2000;343(26):1910-1916.

4. Ghia P, Stamatopoulos K, Belessi C, et al Geographic patterns and pathogenetic implications of IGHV gene usage in chronic lymphocytic leukemia: the lesson of the IGHV3-21 gene. Blood. 2005;105(4):16781685

5. Grever MR, Lucas DM, Dewald GW, et al. Comprehensive assessment of genetic and molecular features predicting outcome in patients with chronic lymphocytic leukemia: results from the US Intergroup Phase III Trial E2997. J Clin Oncol. 2007; 25(7):799-804

6. Hallek M, Fischer K, Fingerle-Rowson G, et al. Addition of rituximab to fludarabine and cyclophosphamide in patients with chronic lymphocytic leukaemia: a randomised, open-label, phase 3 trial. Lancet. 2010; 376(9747):1164-1174

7. Catovsky D, Richards S, Matutes E, et al. Assessment of fludarabine plus cyclophosphamide for patients with chronic lymphocytic leukaemia (the LRF CLL4 Trial): a randomised controlled trial. Lancet. 2007; 370(9583):230-239.

8. Wang L, Lawrence MS, Wan Y, et al. SF3B1 and other novel cancer genes in chronic lymphocytic leukemia. N Engl J Med. 2011; 365(26):2497-2506.

9. Puente XS, Pinyol M, Quesada V, et al Whole-genome sequencing identifies recurrent mutations in chronic lymphocytic leukaemia. Nature. 2011;475(7354):101105.

10. Landau DA, Tausch E, Taylor-Weiner AN, et al. Mutations driving CLL and their evolution in progression and relapse. Nature. 2015;526(7574):525-530

11. Puente XS, Beà S, Valdés-Mas R, et al. Noncoding recurrent mutations in chronic lymphocytic leukaemia. Nature. 2015; 526(7574):519-524.

12. Oscier DG, Rose-Zerilli MJJ, Winkelmann $\mathrm{N}$, et al. The clinical significance of NOTCH1 and SF3B1 mutations in the UK LRF CLL4 trial. Blood. 2013;121(3):468-475.

13. Stilgenbauer S, Schnaiter A, Paschka P, et al.
Gene mutations and treatment outcome in chronic lymphocytic leukemia: results from the CLL8 trial. Blood. 2014;123(21):32473254.

14. Baliakas P, Hadzidimitriou A, Sutton L-A, et al. Recurrent mutations refine prognosis in chronic lymphocytic leukemia. Leukemia. 2015;29(2):329-336.

15. Fabbri G, Rasi S, Rossi D, et al. Analysis of the chronic lymphocytic leukemia coding genome: role of NOTCH1 mutational activation. J Exp Med. 2011;208(7):1389-1401.

16. Weissmann S, Roller A, Jeromin S, et al. Prognostic impact and landscape of NOTCH1 mutations in chronic lymphocytic leukemia (CLL): a study on 852 patients. Leukemia. 2013:27(12):2393-2396.

17. Larrayoz M, Rose-Zerilli MJJ, Kadalayil L, et al. Non-coding NOTCH1 mutations in chronic lymphocytic leukemia; their clinical impact in the UK CLL4 trial. Leukemia. 2017;31(2):510-514.

18. Gupta IV, Jewell RC. Ofatumumab, the first human anti-CD20 monoclonal antibody for the treatment of $\mathrm{B}$ cell hematologic malignancies. Ann N Y Acad Sci. 2012; 126343-126356.

19. Hillmen P, Robak T, Janssens A, et al. Chlorambucil plus ofatumumab versus chlorambucil alone in previously untreated patients with chronic lymphocytic leukaemia (COMPLEMENT 1): a randomised, multicentre, open-label phase 3 trial. Lancet. 2015;385(9980):1873-1883.

20. Rose-Zerilli MJJ, Forster J, Parker $\mathrm{H}$, et al. ATM mutation rather than BIRC3 deletion and/or mutation predicts reduced survival in 11q-deleted chronic lymphocytic leukemia: data from the UK LRF CLL4 trial. Haematologica. 2014;99(4):736-742.

21. Rossi D, Khiabanian H, Spina V, et al. Clinical impact of small TP53 mutated subclones in chronic lymphocytic leukemia. Blood. 2014;123(14):2139-2147.

22. Malcikova J, Stano-Kozubik K, Tichy B, et al. Detailed analysis of therapy-driven clonal evolution of TP53 mutations in chronic lymphocytic leukemia. Leukemia. 2015; 29(4):877-885.

23. Pospisilova S, Sutton L-A, Malcikova J, et al. Innovation in the prognostication of chronic lymphocytic leukemia: how far beyond TP53 gene analysis can we go? Haematologica. 2016;101(3):263-265.

24. Rossi D, Fangazio M, Rasi S, et al. Disruption of BIRC3 associates with fludarabine chemorefractoriness in TP53 wild-type chronic lymphocytic leukemia. Blood. 2012;119(12):2854-2862.

25. Hernández JÁ, Hernández-Sánchez $M$, Rodríguez-Vicente $\mathrm{AE}$, et al. A low frequency of losses in 11q chromosome is associated with better outcome and lower rate of genomic mutations in patients with chronic lymphocytic leukemia. PLoS One. 2015;10(11):e0143073

26. Nadeu F, Delgado J, Royo C, et al. Clinical impact of clonal and subclonal TP53, SF3B1, BIRC3, NOTCH1, and ATM mutations in chronic lymphocytic leukemia. Blood. 2016;127(17):2122-2130.

27. Jeromin $S$, Weissmann $S$, Haferlach C, et al. SF3B1 mutations correlated to cytogenetics and mutations in NOTCH1, FBXW7 MYD88, XPO1 and TP53 in 1160 untreated CLL patients. Leukemia. 2014;28(1):108117.

28. Rossi D, Bruscaggin A, Spina V, et al. Mutations of the SF3B1 splicing factor in chronic lymphocytic leukemia: association with progression and fludarabine-refractoriness. Blood. 2011;118(26):6904-6908.

29. Rossi D, Rasi S, Spina V, et al. Integrated mutational and cytogenetic analysis identifies new prognostic subgroups in chronic lymphocytic leukemia. Blood. 2013; 121(8):1403-1412.

30. Bo MD, Del Principe MI, Pozzo F, et al. NOTCH1 mutations identify a chronic lymphocytic leukemia patient subset with worse prognosis in the setting of a rituximab-based induction and consolidation treatment. Ann Hematol. 2014;93(10): 1765-1774

31. Del Giudice I, Rossi D, Chiaretti S, et al. NOTCH1 mutations in +12 chronic lymphocytic leukemia (CLL) confer an unfavorable prognosis, induce a distinctive transcriptional profiling and refine the intermediate prognosis of +12 CLL Haematologica. 2012;97(3):437-441.

32. Pozzo F, Bittolo T, Arruga F, et al. NOTCH1 mutations associate with low CD20 level in chronic lymphocytic leukemia: evidence for a NOTCH1 mutation-driven epigenetic dysregulation. Leukemia. 2016;30(1):182189.

33. Fürstenau $M$, Hallek $M$, Eichhorst $B$ Sequential and combination treatments with novel agents in chronic lymphocytic leukemia. Haematologica. 2019, 104(11):2144-2154

34. Kater AP, Seymour JF, Hillmen P, et al. Fixed duration of venetoclax-rituximab in relapsed/refractory chronic lymphocytic leukemia eradicates minimal residual disease and prolongs survival: post-treatment follow-up of the MURANO Phase III Study. J Clin Oncol. 2019;37(4):269-277.

35. Moreno C, Greil R, Demirkan F, et al. Ibrutinib plus obinutuzumab versus chlorambucil plus obinutuzumab in first-line treatment of chronic lymphocytic leukaemia (iLLUMINATE): a multicentre, randomised, open-label, phase 3 trial Lancet Oncol. 2019;20(1):43-56. 